\title{
nano dossiers
}

Nr. 005 • Mai 2008

A. Gazsó*, U. Fiedeler, M. Simkó, M. Nentwich

\section{Umwelt- und Gesundheits- auswirkungen von Nanopartikeln - EU-Projekte im 6. Rahmenprogramm}

\section{Zusammenfassung}

Im 6. Forschungsrahmenprogramm investierte die Europäische Kommission mehr als 30 Millionen Euro in die Erforschung von Umwelt- und Gesundheitsauswirkungen von Nanopartikeln (NP) und Nanomaterialien. Während zunächst der Schwerpunkt auf möglichen Gesundheitsauswirkungen gelegen war, wurden in der letzten Zeit auch zunehmend mögliche Umweltauswirkungen thematisiert. Die vier Hauptthemen der Projekte sind:

- Schaffung einer Wissensbasis: Umweltund Gesundheitsauswirkungen von NP (IMPART-NANOTOX); Carbonanotubes und deren Anwendungen (CANAPE); internationale Strategie der Risikobewertung von NP (IMPART-NANOTOX).

- Toxikologie: Zusammenhang zwischen physikochemischen Eigenschaften von NP und ihres möglichen toxischen Potenzials (CELLNANOTOX); Aussagen zur möglichen Toxizizät von Carbonanotubes (CANAPE).

- Zell- und organspezifische Forschung: Interaktionen zwischen NP und lebenden Zellen (NANOINTERACT); Zellmodell zur NP-induzierten Immuntoxizität (DIPNA).

- Arbeits- und Umweltschutz: Charakterisierung von NP, Definition und Beschreibung von Belastungsniveaus in Labors und am Arbeitsplatz (NANOSH); Verfahren zur Risikoanalyse industriell gefertigter NP und Erstellung eines Risikomanagementsystems (NANOSAFE2); kontrollierte Entwicklung von multifunktionalen nanostrukturierten Produkten unter Beachtung des gesamten Lebenszyklus (SAPHIR); Nanotechnologien in Zusammenhang mit Umwelt und Arbeit (NANOCAP).

\section{Einleitung}

Sowohl in der Mitteilung der Europäischen Kommission "Auf dem Weg zu einer europäischen Strategie für Nanotechnologie ${ }^{\prime 1}$ als auch im Europäischen Aktionsplan für Nanotechnologie ${ }^{2}$ („NAnowissenschaften und Nanotechnologien: Aktionsplan für Europa 2005-2009") wird die Notwendigkeit eines sicheren und verantwortungsvollen Umgangs mit den Nanotechnologien betont. Gleichzeitig wurden gegenüber dem 5. Forschungsrahmenprogramm, in dem insgesamt etwa 2,5 Millionen Euro für Forschungsprojekte zu möglichen Umwelt- und Gesundheitsauswirkungen ${ }^{3}$ der Nanotechnologien ausgegeben wurden, die Geldmittel für diesen Bereich auf mehr als das 12-fache (mehr als 30 Millionen Euro) aufgestockt. Ein Anfang 2008 erschienener Bericht der Europäischen Kommission bietet eine Übersicht über viele abgeschlossene und laufende Projekte zu Gesundheitsund Umweltthemen auf europäischer und nationaler Ebene ${ }^{4}$.

Es ist zu erwarten, dass im 7. Rahmenprogramm die Forschungsschwerpunkte der vorangegangenen Rahmenprogramme fortgesetzt und verstärkt werden. ${ }^{5}$ Vom 17. bis 18. April 2008 fand in Brüssel ein Workshop statt, an dem die noch laufenden Projekte des FP7 und die geplanten bzw. bereits genehmigten Projekte des FP7 (wie etwa NanolmpactNet und NanoTest) präsentiert und diskutiert wurden. ${ }^{6}$ Wie wichtig der Europäischen Kommission sicherheits- bzw. risikorelevante Themen der $\mathrm{Na}$ notechnologie sind, zeigt auch die Gründung des Projekts ObservatoryNANO, das im Wesentlichen der Früherkennung von Risiken dient und basierend auf wissenschaftlicher Expertise fundierte Politikberatung zu diesen Themen geben soll. ${ }^{7}$ Eine ähnliche Ausrichtung hat auch das Projekt NanoTrust des Instituts für TechnikfolgenAbschätzung der Österreichischen Akademie der Wissenschaften. ${ }^{8}$
Dieses Dossier gibt eine Übersicht über die derzeit laufenden bzw. kürzlich abgeschlossenen Projekte des 6. Forschungsrahmenprogramms in diesem Bereich. Der Vollständigkeit halber wurden auch zwei Projekte in diese Übersicht aufgenommen, die nur mittelbar im Rahmen ihrer naturwissenschafflichen Aufgabenstellungen auch gesundheits- (CANAPE) bzw. umweltrelevante (POLYSOA) Themen bearbeiten.

\section{CELLNANOTOX (STReP')}

Titel: Cellular interaction and Toxicology with engineered nanoparticles

Leitung: Prof. Rafi Korenstein, Tel Aviv University, Dept. of Physiology \& Pharmacology, Faculty of Medicine, IL-69978 Tel Aviv, Israel

Beginn: 1.11 .2006

Ende: 31.10 .2009 (= 36 Monate)

Projektkosten: 3,65 Mill. $€$

EU-Förderung: 2,6 Mill. $€$

Homepage:

www.fp6-cellnanotox.net/6.html

Kontakt: korens@post.tau.ac.il

Das Ziel des Projekts ist die Aufklärung der Wechselbeziehung zwischen den physikochemischen Eigenschaften von Nanopartikeln (NP) und ihres möglichen toxischen Potenzials auf verschiedene Organe des menschlichen Körpers. Neben der Anwendung konventioneller toxikologischer Methoden werden auch Methoden der Toxikogenomik und moderne Datenverarbeitungstechniken wie Knowledge Discovery from Data (KDD) und Data Mining angewendet.

Zu erwarten sind Ergebnisse bezüglich der Belastung der allgemeinen Bevölkerung und von Arbeitern durch industriell hergestellte, synthetische NP (manufactured nanoparticles, MNP). Des Weiteren sollen verbindliche Aussagen über mögliche Gesund- 
heitsgefährdungen durch MNP getroffen werden, die schließlich zu Empfehlungen zur Regulierung der Anwendung von MNP in diesen Bereichen führen sollen.

Projektpartner sind unter anderem das französische Institut national de la santé et de la recherche médicale (INSERM), das Joint Research Centre in Ispra und die BASF AG, die auch an anderen einschlägigen nationalen und internationalen Forschungsprojekten zur Toxikologie von NP (NanoCare, Nanosafe2, Nano Safety Projekt des HESI/ $\mathrm{ILSI}^{10}$ ) beteiligt sind.

\section{DIPNA (STReP)}

Titel: Development of an integrated platform for nanoparticle analysis to verify their possible toxicity and the eco-toxicity

Leitung: Dr. Antonietta M. Gatti, CNISM (Consortio Naniotale Interuniversitario per le Scienze Fisiche della Materia), Abteilung für Neurowissenschaften, Universität Modena, Italien

Beginn: 1.11.2006

Ende: 31.10 .2009 (= 36 Monate)

Projektkosten: 4,54 Mill. $€$

EU-Förderung: 2,79 Mill. $€$

Österr. Beteiligung: Universität Salzburg, Abt. für Molekularbiologie

(Univ.-Prof. Albert Duschl)

EU-Rückfluss: $247.000 €$

Homepage: www.dipna.eu

Kontakt: gatti@dipna.eu

oder antonietta.gatti@unimore.it

Das Hauptziel dieses Projekts ist die Entwicklung eines Zellmodells zur NP-induzierten Immuntoxizität, das die in vivo Befunde bestmöglich zu erklären in der Lage ist. Dafür werden u. a. In-vitro Tests zur Wechselwirkung zwischen künstlichen Nanopartikeln und Körperzellen durchgeführt. Diese sollen dann die Identifikation der Schlüsselmechanismen der Partikel-Zell-Interaktion unterstützen. Zusätzlich und in weiterer Folge soll ein entsprechendes Zellmodell entwickelt werden, das für die Laborbefunde geeignet ist. Schließlich soll dann dieses Zellmodell auch auf die Felduntersuchungen angewendet und entsprechend angepasst werden.

Die Anforderungen sind zum einen die Entwicklung geeigneter immun- und nanotoxikologischer Methoden und zum anderen die Abschätzung der Auswirkungen von Nanopartikeln auf Einzelzellen als auch die Abschätzung der NP-Belastung in der Arbeitsumgebung.

\section{IMPART (CA ${ }^{11}$ ) Phase 2 bZW. IMPART-NANOTOX}

Titel: Improving the understanding of the impact of nanoparticles on human health and the environment

Leitung Phase 2: Dr. Karl Höhener, TEMAS AG, CH-9320 Arbon, Schweiz ${ }^{12}$

Beginn: 1.2.2005 (Phase 1); 1.6.2007 (Phase 2)

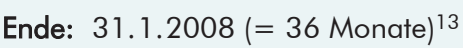

Projektkosten: $741.826 €$

EU-Förderung: $699.913 €$

Homepage: www.impart-nanotox.org

Kontakt: karl.hoehener@temas.ch

\section{NANOTOX (ursprünglich, SSA $\left.^{14}\right)^{15}$}

Titel: Investigative support for the elucidation of the toxicological impact of nanoparticles on human health and the environment

Leitung: Dr. Mark Pullinger,

Chalex Research Ltd., Torquay, UK

Beginn: 1.2.2005

Ende: 31.1.2007 (24 Monate)

Projektkosten: $408.544 €$

EU-Förderung: $399.894 €$

Homepage: -

Kontakt:

Das Projekt IMPART sollte ursprünglich Möglichkeiten erarbeiten, die verhindern sollen, dass die Erkenntnisse über die Gesundheitsund Umweltauswirkungen von Nanopartikeln hinter den technologischen Entwicklungen und Fortschritten zurück bleiben (so die Eigenbeschreibung) ${ }^{16}$.

IMPART-NANOTOX ist aus einer Zusammenlegung der beiden Ausgangsprojekte IMPART und NANOTOX entstanden, da ihre Aufgabenstellungen als komplementär erachtet wurden. Die entsprechende aktuelle Homepage $^{17}$ enthält Informationen über beide Ausgangsprojekte und den aktuellen Projektcluster. Außerdem bietet sie einen Zugang zu einer Literatur-Datenbank zu Gesundheits- und Umweltauswirkungen von Nanopartikeln im Umfang von etwa 350 Einträgen. ${ }^{18}$ Eine Homepage zum abgeschlossenen Projekt NANOTOX existiert nicht.
Das Konsortium unter der Koordination der Schweizer TEMAS AG besteht in Phase 2 aus 19 Partnern, darunter auch die Eidgenössische Materialprüfanstalt (EMPA) und der Verein deutscher Ingenieure (VDI).

Die Aufgaben des Projekts sind der regelmäßige Review der neuesten wissenschaftlichen Arbeiten zu Umwelt- und Gesundheitsauswirkungen von Nanopartikeln. Außerdem sollen entsprechende Bemühungen auf nationaler und internationaler Ebene koordiniert werden, um gemeinsame Strategien im Umgang mit der Risikobewertung von NP zu entwickeln und Fördergeber bei der Gestaltung zukünftiger Forschungsprogramme zur Nanotoxikologie zu beraten.

Ende März 2008 hat in Zürich eine Begutachtung in der Mitte der Laufzeit stattgefunden. Das Projekt wird im Herbst 2008 auf allen großen europäischen Konferenzen zum Thema Nanotoxikologie vertreten sein (Nanotox2008, NanoEurope 2008, Eurotox 2008, Nanosafe 2008). Enge Kontakte bestehen zum Nanoforum der Europäischen Kommission ${ }^{19}$, SHAPE-RISK, Nanonet ${ }^{20}$ und NanoTox $^{21}$, einem Online-Forum zur Toxizität von industriell hergestellten Nanomaterialien.

\section{NANOTOX (FP6-Marie Curie Stipendium) $^{22}$}

Titel: Nanoparticle characterization and toxicity

Leitung: Dr. Kjeld Alstrup Jensen, National Research Centre for the Working Environment, Denmark Beginn: 1.12.2006 23

Ende: 30.11 .2008 (24 Monate)

Projektkosten: $180.134 €$

EU-Förderung: $180.134 €$

Homepage: -

Kontakt:

Am dänischen Institut für Arbeitssicherheit gemeinsam mit der Universität Kopenhagen soll der Einfluss der physikochemischen Eigenschaften von Nanopartikeln (Größe, Form, Größe der Oberfläche, Löslichkeit und chemische Zusammensetzung) auf deren Toxizität untersucht werden. Als Versuchsmodell werden menschliche Hautzellen (Epithelzellen) verwendet. Die mögliche Toxizität wird an Hand verschiedener Parameter gemessen, die entzündliche Reaktionen und oxidativen Stress (Produktion freier Radikale, Konzentration entzündungsfördernder Zytokine), aber auch programmierten Zelltod, DNA-Schäden etc. anzeigen. 


\section{NANOINTERACT (STReP)}

Titel: Development of a platform and toolkit for understanding interactions between nanoparticles and the living world

Leitung: Prof. Kenneth Dawson,

University College Dublin, National University of Ireland, Conway Institute of Biomolecular and Biomedical Research

Beginn: 1.1.2007

Ende: 31.12.2009 (36 Monate)

Projektkosten: 4,62 Mill. $€$

EU-Förderung: 3,3 Mill. $€$

Homepage: www.nanointeract.net

Kontakt: info@nanointeract.net

Nanolnteract ist ein von der Europäischen Kommission gefördertes Projekt, das der Aufklärung der Wechselwirkungen von synthetischen Nanopartikeln mit lebenden Systemen gewidmet ist. Das Projekt besteht aus 9 Partnern aus der akademischen Forschung (darunter einer aus den USA: Rice University), zwei Europäischen Forschungszentren und 6 Industriepartnern (u. a. Intel und l'Oréal).

Im Zentrum steht die Untersuchung und Beschreibung der Interaktionen zwischen $\mathrm{Na}$ nopartikeln und lebenden Zellen, beginnend mit dem Eintritt der Partikel in den lebenden Organismus, ihrem Transport, der Art ihrer Aufnahme und Akkumulation in Geweben und Organen und der Klärung der Frage ihrer Wechselwirkung mit der Einzelzelle. Besonders soll der Frage nachgegangen werden, ob und wie synthetische NP in die Zelle eindringen und welche Eigenschaften der NP (Größe, Form, Oberfläche etc.) dieses Verhalten beeinflussen. Insbesondere sollen durch Protokollierung und Standardisierung von Experimenten eine Grundlage für die Reproduzierbarkeit entsprechender Untersuchungsergebnisse geschaffen werden.

Die Homepage bietet eine allgemeine Einführung in die Voraussetzungen und allgemeinen Fragestellungen des Projekts. Nähere Informationen zu Ergebnissen, Profilen der Industriepartner und Kontaktdaten sind nur über eine Nutzerberechtigung zu erhalten.

\section{NANOSH (STReP)}

Titel: Inflammatory and genotoxic effects of engineered nanomaterials

Leitung: Prof. Kai Savolainen, Finnish Institute of Occupational Health

Beginn: 1.11.2006

Ende: 31.10 .2009 (36 Monate)

Projektkosten: 4 Mill. $€$

EU-Förderung: 2,4 Mill. $€$

Homepage:

www.ttl.fi/Internet/partner/Nanosh/

Kontakt: Kai.Savolainen@ttl.fi

Die Hauptziele dieses Projekts sind die Charakterisierung von Nanopartikeln und die Definition und Beschreibung von Belastungsniveaus in Labors und am Arbeitsplatz. Speziell sollen die Gentoxizität, die Immuntoxizität und andere Effekte auf den menschlichen Körper bestimmt werden. Im Rahmen dieses Projekts sollen daher entsprechende Nachweis- und Analysemethoden entwickelt werden.

\section{PARTICLE_RISK (STReP)}

Titel: Risk assessment of exposure to particles

Leitung: Dr. Lang Tran, Institute of Occupational Medicine, Quantitative Toxicology, Riccarton, UK

Beginn: 1.6.2005

Ende: 31.5. 2008 (36 Monate)

Projektkosten: 1,12 Mill. $€$

EU-Förderung: $799.576 €$

Homepage: www.iom-world. org/ research/nanoparticles.php

Kontakt: lang.tran@iom-world.org

Dieses Projekt hat zum Ziel, Methoden zur Bewertung möglicher Gefahren durch neuartige Partikel (i. w. S.) zu entwickeln. Es behandelt daher nicht nur synthetische nanoskalige Partikel, sondern auch größere Partikel und solche, die in Verbrennungsprozessen entstehen (",combustion engineered particles"). Einem Teil der Untersuchungen liegt die Annahme zu Grunde, dass nanoskalige Partikel u. a. die Bildung von Arteriosklerose fördern können.

Zunächst soll eine Datenbank erstellt werden, die die charakteristischen Eigenschaften von neuartigen Partikeln beinhaltet (CNT/ carbon nanotubes, Fulleren, quantum dots,
Gold- und Kohlenstoffpartikel etc.). Daneben sollen Methoden entwickelt werden, mit denen NP in lebendem Gewebe nachgewiesen und quantifiziert werden können. In Tierversuchen (Mäuse) und durch In-vitro-Untersuchungen an kultivierten Zellen soll die Toxizität von NP bestimmt werden. In einem weiteren Schritt wird dann in diesem Projekt der Versuch unternommen, aus den gewonnenen Erkenntnissen eine Aussage zum Risiko für den Menschen zu treffen.

\section{NANOSAFE2 (IP)}

Titel: Safe production and use of nanomaterials

Leitung: Dr. Frédéric Schuster,

Commissariat à l'energie atomique (CEA), Grenoble, Frankreich

Beginn: 1.4.2005

Ende: 31.3.2009 (48 Monate)

Projektkosten: 12,4 Mill. $€$

EU-Förderung: 7 Mill. $€$

Homepage: www.nanosafe.org

Kontakt: frederic.schuster@cea.fr

Das Projekt Nanosafe2 besteht aus 24 Partnern aus Wissenschaft und Industrie, die von der französischen Atomenergiebehörde koordiniert werden. Diese ist auch technischer Koordinator im Projekt SAPHIR (s. d.). Im Wesentlichen werden 2 Ziele verfolgt, nämlich einerseits ein Verfahren zur Risikoanalyse industriell gefertigter Nanopartikel zu entwickeln und andererseits darauf aufbauend ein entsprechendes Risikomanagementsystem für den sicheren Umgang mit NP in der industriellen Produktion abzuleiten. Dafür sollen zunächst bestimmte Referenzpartikel ausgewählt werden.

In das Risiko- und Sicherheitsmanagement sollen Präventivmaßnahmen integriert werden, die mit verschiedenen Interessensvertretern zusammen erarbeitet werden. In weiterer Folge sollen Materialien entwickelt werden, die zur Aus-, Fort- und Weiterbildung genützt werden können.

Im Wesentlichen werden 2 Anlassfälle genaver untersucht: (1) mögliche Explosionen bei Herstellungsprozessen und (2) Auswirkungen von NP auf die menschliche Gesundheit bei chronischer Exposition.

Die Forschungsarbeiten im Rahmen dieses Projekts sollen schließlich zur Entwicklung neuen Detektions- und Charakterisierungstechniken für synthetische Nanopartikel in Luft und flüssigen Medien führen. Weiters 
sollen auch toxikologische Untersuchungen zur Identifizierung von Gefahrenpotenzialen und Dosis-Wirkungsbeziehungen durchgeführt werden. Der Schwerpunkt liegt dabei auf Carbon Nanotubes, Carbon Black, Zinkoxid und Siliziumdioxid. Außerdem sollen sichere Produktionstechnologien entwickelt und vorgeführt werden. In einem darüber hinausreichenden Schritt sollen auch gesellschaftsrelevante Aspekte in die Risikobewertung aufgenommen werden und schließlich auch in die Standardisierung und Gesetzgebung einfließen.

Das Projekt publiziert einen Newsletter, der auf der Homepage zugänglich ist. Im Jänner 2008 wurde ein erster sogenannter "Dissemination Report" veröffentlicht, der eine kurze allgemeinverständliche Zusammenfassung der wichtigsten Ergebnisse des Projekts bietet.

\section{NANOTRANSPORT (SSA)}

Titel: The behaviour of aerosols released to ambient air from nanoparticle manufacturing - A pre-normative study

Leitung: Dr. Qinglan Wu, Det Norske Veritas AS (DNV), Hoevik, Norwegen

Beginn: 1.9.2006

Ende: 29.2.2008 (18 Monate)

Projektkosten: $450.000 €$

EU-Förderung: $450.000 €$

Homepage:

research.dnv.com/nanotransport/

Kontakt: Qinglan.Wu@dnv.com

Das Projekt NanoTransport beschäftigt sich mit dem Verhalten von Aerosolen in der Umgebungsluft von Arbeitsplätzen von Betrieben, die Nanomaterialien herstellen bzw. verarbeiten. Die Hauptaufgaben bestehen zunächst in der Definition realistischer Testbedingungen hinsichtlich der Charakterisierung der verwendeten Aerosole, die für nanotoxikologische Untersuchungen verwendet werden sollen. Danach sollen entsprechende technische Kontrollsysteme (Feinstaub-Messgeräte, Messgeräte für Emissionen und technische Gase) auf ihre Effizienz getestet und gegebenenfalls kalibriert und zertifiziert werden. Die Ergebnisse dieses Projekts dienten als Grundlage zur Erstellung für Empfehlungen an die Europäische Kommission ${ }^{24}$. Insbesondere wird erwartet, dass die in diesem Projekt gewonnenen Erkenntnisse zur Entwicklung von standardisierten Test-Aerosolen dienen.

\section{NANOCAP (CA)}

Titel: Nanotechnology capacity building NGOs

Leitung: Drs. Jacques Cornelis van Broekhuizen, IVAM UvA BV, Amsterdam, Niederlande

Beginn: 1.9.2006

Ende: 31.8.2009 (36 Monate)

Projektkosten: 1,31 Mill. $€$

EU-Förderung: 1,31 Mill. $€$

Österr. Beteiligung: ppm Forschung und Beratung, Linz (Dr. Günther Kittel)

EU-Rückfluss: $70.080 €$

Homepage: www.nanocap.eu

Kontakt: pvbroekhuizen@ivam.uva.nl

Österr. Homepage:

www.ppm.at/nanoinfo/

Kontakt in Österreich: nanoinfo@ppm.at

NanoCap ist ein Netzwerk von 16 Partnern. Beteiligt sind neben dem niederländischen Koordinator je fünf Institutionen aus Wissenschaft, Gewerkschaften bzw. gewerkschaftsnahen Einrichtungen und Umwelt-NGOs.

Ziel dieses Projekts ist eine Verbesserung des Verständnisses von Nanotechnologien in Zusammenhang mit Umwelt und Arbeit bei allen beteiligten Akteuren, die Information der Mitglieder von Gewerkschaften, NGOs und der interessierten Öffentlichkeit zu umwelt-, gesundheits- und sicherheitsrelevanten Problemen der Nanotechnologien und die Unterstützung der Gewerkschaften und NGOs bei der Formulierung ihrer politischen Positionen zu diesen Themen. Der Interessensschwerpunkt des österreichischen Partners liegt auf dem Gebiet des Arbeitnehmerinnenschutzes.

Bisher haben drei von vier geplanten Arbeitstagungen des Projektteams stattgefunden. Die vierte Tagung zum Thema "Ethische Fragen und Strategien" ist für September 2008 geplant. Im April 2009 soll dann eine öffentliche Abschlusskonferenz stattfinden.

\section{SAPHIR (IP)}

Dieses Projekt vereinigt mehr als zwanzig Partner unter französischer Führung, wobei die Funktionen getrennt sind. Das Projektmanagement liegt bei der Firma CILAS, einem mittelgroßen französischen Betrieb zur Entwicklung und Herstellung optischer Beschichtungen und nanostruktierter Oberflächen ${ }^{25}$ für zivile und militärische Anwendun- gen. Die technische Koordination hat die französische Atomenenergiebehörde (CEA) übernommen, wodurch das Projekt SAPHIR mit Nanosafe2 verbunden ist.

Das Hauptziel dieses auf vier Jahre anberaumten Projekts liegt in der sicheren und kontrollierten Entwicklung und Herstellung von multifunktionalen nanostrukturierten Hochtechnologie-Produkten, wobei der gesamte Lebenszyklus dieser Produkte betrachtet wird.

Im Oktober 2007 hat eine zweitägige Konferenz mit dem Titel "Innovating industrial processes applied to new materials" an der kanadischen Sherbrooke University stattgefunden. Teilgenommen haben die Partner aus dem Projekt SAPHIR und 36 kanadische Institutionen aus Industrie und Wissenschaft.

Titel: Controlled production of high tech multifunctional products and their recycling

Leitung: Christophe Goepfert,

Compaignie industrielle des lasers

(CILAS), Frankreich

Beginn: 1.10 .2006

Ende: 30.9.2010 (48 Monate)

Projektkosten: 15,79 Mill. $€$

EU-Förderung: 8,1 Mill. $€$

Homepage:

www. saphir-project.eu/index. php? $\mathrm{id}=48$

Kontakt: goepfert@cilas.com

\section{POLYSOA (STREP)}

Titel: Polymers in Secondary Organic Aerosols (NEST-2003-2 Insight activities)

Leitung: Urs Baltensperger,

Paul Scherrer Institut, Laboratoriums für Atmosphärenchemie, Schweiz

Beginn: 1.10.2005

Ende: 30.9.2007 (24 Monate)

Projektkosten: 1,13 Mill. $€$

EU-Förderung: $795.000 €$

Österr. Beteiligung:

Prof. Dr. Hans Puxbaum, Institute for Chemical Technologies and Analytics, Vienna University of Technology

Homepage: polysoa.web.psi.ch

Kontakt: urs.baltensperger@psi.ch

Kontakt in Österreich:

hpuxbaum@mail.zserv.tuwien.ac.at 
Im Rahmen dieses Projekts sollen verschiedene analytische Methoden zur Messung von «high molecular weight compounds» in Aerosolen entwickelt und angewendet werden. Damit sollen diese Verbindungen chemisch-physikalisch charakterisiert werden. Obwohl dieses Programm nicht spezifisch auf Nanopartikel Bezug nimmt, könnten diese Methoden Anhaltspunkte für die Messung von Nanopartikeln in der Umgebungsluft liefern. Die Technische Universität Wien ist Partner in diesem Projekt.

\section{CANAPE (IP)}

Titel: Carbon Nanotubes for Applications in Electronics, Catalysis, Composites and Nano-Biology

Leitung: John Robertson, University of Cambridge, United Kingdom

Beginn: 1.6.2004

Ende: 31.5 .2008 (48 Monate)

Projektkosten: 8,8 Mill. $€$

EU-Förderung: 6,4 Mill. $€$

Homepage: www.canapeweb.com

Kontakt: jr@eng.cam.ac.uk

Das Projekt versucht das fundamentale Wissen über Carbonanotubes (CNT) und deren Anwendungen in verschiedenen Bereichen (Chemie, Elektronik, Verbundwerkstoffe) zusammenzutragen. Ziel ist die Produktion von CNT durch "chemical vapour deposition" (CVD) in großen Mengen (Tonnen/Jahr).

Im Rahmen des 6. Arbeitspakets "Health" werden CNT auf ihre Toxizität untersucht. Die Studie versucht dabei herauszufinden, wie die Toxizität von der Länge und vom Durchmesser der CNT abhängt und ob die Toxizität möglicherweise von katalytischen Rückständen abhängt. Weiter soll abgeklärt werden, ob die toxischen Effekte mit standardisierten Tests nach ISO erfasst werden können. Die Bestimmung eines NOEC 26 für Nanotubes (ohne weitere Angaben zu Medium, Organismus etc.), die Identifizierung von für die Toxizität kritischen Eigenschaften sowie weitere Fragestellungen zur Toxizität von CNT werden bearbeitet. Zu den anderen Arbeitspaketen werden mit der Begründung der Geheimhaltung keine detaillierten Informationen angegeben. Die Empa ist ein Projektpartner im Arbeitpaket 6 zu etwaigen Gesundheitsauswirkungen der CNT.

\section{Fazit}

Die Erforschung der Gesundheits- und Umweltauswirkungen von Nanopartikeln und Nanomaterialien hat ein großes Potenzial, sowohl auf europäischer als auch auf nationaler Ebene, wobei der Schwerpunkt nach wie vor auf humantoxikologischer Forschung liegt. Ökotoxikologische Fragestellungen rücken aber zunehmend in den Vordergrund. Das Engagement österreichischer Institutionen in internationalen Projekten zu sicherheits- und risikorelevanten Fragestellungen ist zu begrüßen und sollte in $\mathrm{Zu}$ kunft verstärkt werden.

\section{Anmerkungen und Literaturhinweise}

' COM(2004)338 ftp://ftp.cordis.europa.eu/ pub/nanotechnology/docs/

nano_com_de_new.pdf.

2 COM(2005)243 ftp://ftp.cordis.europa.eu/ pub/nanotechnology/docs/ action_plan_brochure_de.pdf.

3 Im Englischen üblicherweise mit dem Akronym $\mathrm{EHS}$ (environmental, health and safety issues) bezeichnet.

4 European Commission (2008): EU nanotechnology R\&D in the field of health and environmental impact of nanoparticles. Compiled by Pilar Aguar and José Juan Murcia Nicolás, Unit G4 Nano and Converging Sciences and Technologies European Commission, Research DG; 28 January 2008.

5 Im ersten Call vom 22. Dezember 2006 gibt es jedenfalls einige unmittelbar relevante Bereiche, u.z. (2) NMP-2007-1.3-1 Specific, easyto-use portable devices for measurement and analysis, (2) NMP-2007-1.3-2 Risk assessment of engineered nanoparticles on health and the environment; (3) NMP-2007-1.3-3 Scientific review of the data and studies on the potential impact of engineered nanoparticles on health, safety and the environment; (4) NMP2007-1.3-4 Creation of a critical and commented database on the health, safety and environmental impact of nanoparticles; (5) NMP2007-1.3-5 Coordination in studying the environmental, safety and health impact of engineered nanoparticles and nanotechnology based materials and products; (6) HEALTH2007-1.3-4: Alternative testing strategies for the assessment of the toxicological profile of nanoparticles used in medical diagnostics (koordiniert mit NMP-2007-4.1.3-2/4.4-4); (7) voon speziellem Interesse ist auch NMP-20071.3-2 Risk assessment of engineered nanoparticles on health and the environment.

6 Workshop on research projects on the safety of nanomaterials: reviewing the knowledge gaps. Brussels 17-18 April 2008. Draft Agenda. ftp://ftp.cordis.europa.eu/pub/nanotechnology/docs/agenda final.pdf.

7 cordis.europa.eu/fetch? CALLER $=$ FP7 NEWS\&ACTION $=$ D\&DOC $=1 \& C A T=$ NEW'S\&QUERY $=0119 \mathrm{e} 7968 c 8 d: 884 c: 6020$ b98d\&RCN $=29419$.

8 nanotrust.ac.at.

9 Specific targeted research project.
10 Health and Environmental Sciences Institute (HESI) des International Life Science Institute (ILSI) in Washington/DC (www.hesiglobal.org).

11 Coordination action.

12 Ursprünglich war die Projektleitung bei Chalex Research in Torquay/UK. Im Cordis project fact sheet ist als Projektleiter ein Dr. Mark Pullinger angeführt. Der Link auf der CORDISÜbersichtsseite (cordis.europa.eu/nanotech nology/src/pressroom_projects_nmp6.htm) führt jedoch bereits auf die Webseite des Projekts IMPART-NANOTOX.

13 Es bestehen erhebliche Abweichungen in den Angaben auf der Projekthomepage und dem EU-Datenblatt (CORDIS fact sheet). Das Enddatum ist im EU-project fact sheet mit 31.1. 2008 angegeben (Projektlaufzeit wäre demnach 36 Monate), im CORDIS-Bericht allerdings sind 42 Monate angegeben. Im CORDIS fact sheet ist IMPART (= Phase 1 von IMPART-NANOTOX) mit "completed” gekennzeichnet. Die aktuelle Projekthomepage IMPART-NANOTOX verzeichnet allerdings noch bis in den November 2008 Veranstaltungen, die mit Impart zu tun haben.

14 Specific support action.

15 Es gibt keine Hinweise darauf, dass NANOTOX ein separates Projekt ist wie sowohl im CORDIS-Bericht als auch Im CORDIS-project fact sheet angegeben ist. Es scheint sich vielmehr um zwei unterschiedliche Projekte mit demselben Akronym zu handeln, nämlich einerseits um eine Marie-Curie-Aktivität der Uni Kopenhagen (It. CORDIS-Bericht von Jänner 2008, Finanzierungsvolumen etwa 180.000 Euro) und andererseits ein eigenes Projekt der Aktionslinie NMP-2002-3.4.1.5-1 „Roadmaps für nanotechnology". Als Kontaktperson ist Mark Pullinger von Chalex Research in Torquay/UK angegeben. Eine entsprechende Projekt-Homepage dazu besteht nicht. Auch der Verweis von der Datenbank des Woodrow Wilson Center (nanotechproject.org) ist ein blind link. Chalex Research kommt in Phase 2 des Impart-Nanotox Projekts nicht mehr vor.

16 "The primary aim of this CA is to prevent knowledge of the health and environmental implications of nanoparticles from lagging behind the technological advances."

17 www.impart-nanotox.org.

18 Erreichbar über www.impart-nanotox.org/ database.html bzw. direkt über die Homepage des Koordinators TEMAS (www.temas.ch/Impart/ImpartProj.nsf/fmPubs ByType?ReadForm\&count=12\&lang=en). 
19 www.nanoforum.org.

20 www.nanonet.org.uk.

21 www.nanotox.info.

22 Laut CORDIS-Bericht vom 28.1.2008.

23 Auf dem entsprechenden CORDIS fact sheet steht allerdings als Anfangsdatum der 18. 10. 2006 und als Enddatum der 17. 10. 2008. Siehe cordis.europa.eu/fetch?CALLER= FP6 PROJ\&ACTION $=$ D\&DOC $=1 \& C A T=$ PROJ \&QUERY $=1194423998403 \& R C N=$ 83326.

24 research.dnv.com/nanotransport/ NANOTRANSPORTdownload/Nanotranport $\% 20$ Recomm\%20\%20FINAL.pdf.

25 www.cilas.com.

26 NOEL (NOEC) = No observed effect level (concentration). Der NOEL (NOEC) ist die höchste Dosis (Expositionskonzentration), bei der keine signifikanten Effekte (keine Abweichungen von der biologischen Norm) beobachtet wurden. Es ist die maximale nicht wirksame Dosis. NOEL- (NOEC-)Werte werden aus längerfristigen Prüfverfahren ermittelt. Signifikante Effekte sind Veränderungen, die als Hinweis auf eine Störung der Lebensabläufe zu werten sind wie z. B. leichte, nicht unbedingt toxische Veränderungen des Blutbildes.

\section{IMPRESSUM:}

Medieninhaber: Österreichische Akademie der Wissenschaften; Juristische Person öffentlichen Rechts (BGBI 569/1921 idF BGBI I 130/2003); Dr. Ignaz Seipel-Platz 2, A-1010 Wien

Herausgeber: Institut für Technikfolgen-Abschätzung (ITA); Strohgasse 45/5, A-1030 Wien;

www.oeaw.ac.at/ita

Erscheinungsweise: Die NanoTrust-Dossiers erscheinen unregelmäßig und dienen der Veröffentlichung der Forschungsergebnisse des Instituts für Technikfolgen-Abschätzung im Rahmen des Projekts NanoTrust. Die Berichte werden ausschließlich über das Internetportal „epub.oeaw” der Öffentlichkeit zur Verfügung gestellt: epub.oeaw.ac.at/ita/nanotrust-dossiers/

NanoTrust-Dossier Nr. 005, Mai 2008: epub.oeaw.ac.at/ita/nanotrust-dossiers/dossier005.pdf

ISSN: 1998-7293 